\title{
CD57 ratio as a convenient and useful immunological and prognostic parameter for stage IV carcinoma
}

\author{
JUNJI AKAGI ${ }^{1}$ and HIDEO BABA ${ }^{2}$ \\ ${ }^{1}$ Department of Surgery, Tamana Regional Health Medical Center, Tamana, Kumamoto 865-0005; \\ ${ }^{2}$ Department of Gastroenterological Surgery, Graduate School of Medical Sciences, \\ Kumamoto University, Kumamoto 860-8556, Japan
}

Received June 14, 2017; Accepted November 30, 2017

DOI: $10.3892 / \mathrm{ol} .2018 .8451$

\begin{abstract}
Cluster of differentiation (CD)8+CD57+ T cells are derived through the $\mathrm{CD} 8+\mathrm{T}$ cell-differentiation signaling pathway from early differentiated CD27+CD8+CD57-T cells (early-CD8+ T cells) to terminal-differentiated CD27-CD8+CD57+ $\mathrm{T}$ cells (terminal-CD8+ $\mathrm{T}$ cells) via intermediate-differentiated CD27+CD8+CD57+ T cells (intermediate-CD8+ T cells). The increase of CD8+CD57+ $\mathrm{T}$ cells in the peripheral blood of patients with cancer has been associated with prognosis, which suggests their suitability as a candidate immunological marker. The present study investigated the association of these CD57-related CD8+ $\mathrm{T}$ cell populations in the peripheral blood of 100 Stage IV cancer patients with progression-free survival (PFS), using a Cox regression model. Univariate analysis indicated that early- and intermediate-CD8+ T cells were associated with shorter PFS, whereas terminal-CD8+ T cells were associated with longer PFS. A strong inverse correlation was observed between early- and terminal-CD8+ T cells, and multivariate analysis demonstrated that the CD57 ratio (terminal-CD8+ $\mathrm{T}$ cells/early-CD8+ T cells) was a more significant independent prognostic factor compared with early- or terminal-CD8+ T cells. Patients with a higher CD57 ratio had a significantly longer PFS compared with those with a lower CD57 ratio, in whom terminal-CD8+ T cells were supposed to be predominant. Conversely, results indicated inhibition of the CD8+ $\mathrm{T}$ cell differentiation signaling pathway in patients with a low CD57 ratio, which lead to a predominance of early-CD8+ $\mathrm{T}$ cells, a characteristic of immunosuppressive cells. The present findings suggested that the CD57 ratio appears to be a powerful immunological prognostic parameter obtained from
\end{abstract}

Correspondence to: Dr Junji Akagi, Department of Surgery, Tamana Regional Health Medical Center, 2172 Tamana, Tamana, Kumamoto 865-0005, Japan

E-mail: jnjakagi@gmail.com

Key words: CD8+CD57+ T cells, CD8+ T cell differentiation, stage IV cancer, prognosis the peripheral blood, precisely reflecting the state of CD8+ T cell-differentiation.

\section{Introduction}

Monitoring the immunological status of cancer patients is an important component of treatment decision and outcome, which requires establishing useful immunological parameters that can be easily and non-invasively obtained from the peripheral blood and that reflect both prognosis and the immunological state. In the search for such markers, we previously investigated the association of immunological parameters such as natural killer cells, Th1 cells, Th2 cells, Th1/Th2 balance, Treg cells, CD57+ T cells, and CD56+ T cells with progression-free survival (PFS) in patients with advanced gastric carcinomas. Unexpectedly, we found that CD57+ $\mathrm{T}$ cells in the peripheral blood of advanced gastric cancer patients were an independent poor prognostic factor (1). A similar result was previously reported in patients with advanced renal cell carcinoma, in that those who harbored higher percentages of CD8+CD57+ lymphocytes in the peripheral blood had shorter overall survival (OS) (2). Moreover, Focosi et al (3) reported that CD57+ expression in $\mathrm{T}$ lymphocytes is a useful marker for measuring functional immune deficiency in patients with autoimmune disease, infectious disease, and cancers. These reports suggest that CD8+CD57+ T cells play an immunosuppressive role among the $\mathrm{CD} 8+\mathrm{T}$ cell population. Treatment with interferon-alpha (IFN- $\alpha$ ) tends to increase the generally low pre-treatment counts of CD8+CD57+ T cells (4). Accordingly, melanoma patients with a lower level of CD8+CD57+ T cells in the peripheral blood before treatment with IFN- $\alpha$ had longer survival longer than those with a higher level of CD8+CD57+ $\mathrm{T}$ cells (5), which indicates that the cytotoxic subsets of the CD8+CD57+ $\mathrm{T}$ cell population may predominate in melanoma patients. Thus, CD8+CD57+ T cell populations appear to be composed of both cytotoxic and immunosuppressive subsets. In the CD8+ T cell differentiation pathway, CD8+CD57+ $\mathrm{T}$ cells are regarded as intermediate-CD8+ T cells (CD27+CD8+CD58+ T cells), which then differentiate to terminal-CD8+ T cells (CD27-CD8+CD57+ T cells). The differentiation of intermediate-CD8+ T cells toward cytotoxic terminal-CD8+ T cells is inhibited by transforming growth 
factor-beta, interleukin-10, and programmed cell death-1 in cancer patients, where the accumulation of incompletely differentiated CD8 $+\mathrm{T}$ cells (likely intermediate-CD8 $+\mathrm{T}$ cells) leads to the uncontrolled progression of malignant cells $(1,6,7)$. Thus, the predominance of cytotoxic over immunosuppressive CD8+CD57+ T cells may be dependent on the state of CD8+ T cell differentiation. These findings suggest that it is important to monitor the dynamic state of $\mathrm{CD} 8+\mathrm{T}$ cell differentiation in cancer patients; that is, to determine which CD8+ $\mathrm{T}$ cell population is predominant in the differentiation pathway.

Therefore, in this study, we investigated the association of each population in the CD8+ T cell differentiation pathway with PFS in a series of patients with various types of cancers in an attempt to identify the parameter that best reflects the dynamic state of CD8+ T cell differentiation. The results of this preliminary analysis are expected to provide new clues for understanding the immunological status of cancer patients, and improving treatment decisions.

\section{Materials and methods}

Patients, sample collection, and processing. All participants provided written informed consent before enrollment. The study protocol was approved by the institutional review board at each participating center (Department of Gastroenterological Surgery, Graduate School of Medical Sciences, Kumamoto University). All methods and procedures associated with this study were conducted in accordance with the Good Clinical Practice guidelines and conformed to the ethical principles of the Declaration of Helsinki and local laws. A total of 100 patients diagnosed with Stage IV carcinomas according to the unified TNM classification (8) at Tamana Regional Health Medical Center between July 2013 and March 2016 were enrolled in the study. The patients (45 men and 55 women) ranged in age from 28 to 96 years (mean $69.0 \pm 13.2$ years). The primary organs affected by cancer in these patients were the stomach (9), colon (23), pancreas (10), liver (6), bile duct (11), lung (14), breast (9), thyroid (6), ovary (8) and others (carcinoma of unknown primary) (4). We collected $10-\mathrm{ml}$ peripheral blood samples from all eligible patients before treatment; patient eligibility was determined by the presence of histologically and clinically diagnosed carcinomas.

Antibodies and flow cytometric analysis (FACS). The following reagents were used for FACS: fluorescein isothiocyanate (FITC)-conjugated anti-CD3 (HIT3a), FITC-conjugated anti-CD57 (NK-1), phycoerythrin (PE)-conjugated anti-CD27 (M-T271), PE-conjugated anti-FOXP3 (259D/C7), PE-conjugated anti-CD57 (NK-1), and anti-CD8 PE-Cy7 (RPA-T8) (BD Biosciences, San Jose, CA, USA). The cells were analyzed on a BD FACSCalibur flow cytometer and evaluated with BD CellQuest software. These data were used to divide the patients into two groups for each parameter based on the proportions of early-CD8+ $\mathrm{T}$ cells, intermediate-CD8+ $\mathrm{T}$ cells, terminal-CD8+ $\mathrm{T}$ cells, and the CD57 ratio (terminal-CD8+ T cells/early-CD8+ $\mathrm{T}$ cells) to determine the independent contributions of each marker for reflecting PFS.

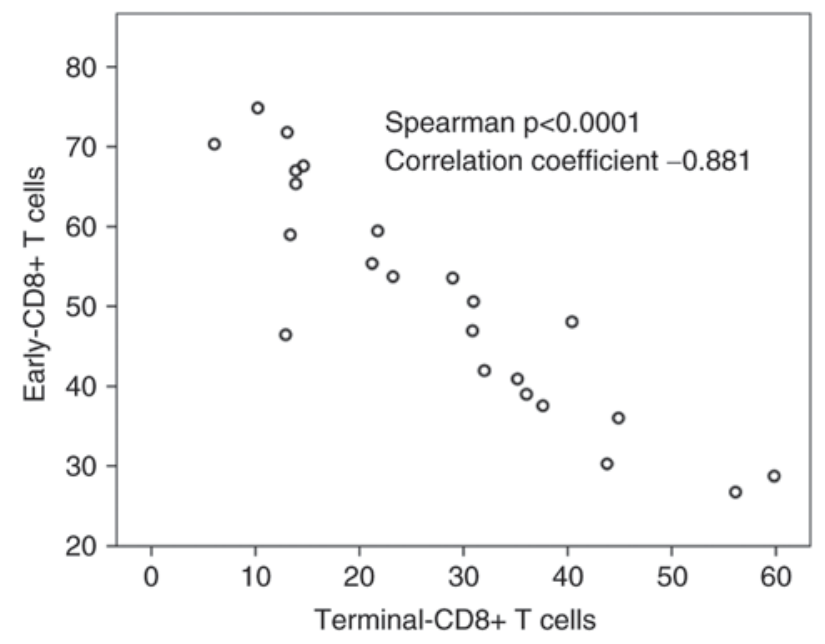

Figure 1. Correlation between early-CD8+ T cells and terminal-CD8+ $\mathrm{T}$ cells. There is a significant reverse correlation between them (Spearman correlation coefficient $=-0.919, \mathrm{P}<0.0001)$.

Study endpoints and assessments. The primary endpoint of this study was PFS. PFS was measured from the date of randomization to the first recurrence or death regardless of the cause. Tumor assessments were performed using dynamic computed tomography or magnetic resonance imaging every 3 months from the baseline for 24 months and every 3-6 months thereafter. All scans at each site were reviewed by two independent and blinded radiologists, each with more than 5 years of experience. In cases of discordance, a third independent experienced radiologist reviewed the images to reach a consensus. Adverse events were classified and graded every 2 months according to the Common Terminology Criteria for Adverse Events (v3.0; National Cancer Institute, Bethesda, MD, USA) from the time of consent until the end of the study or drop-out, continuing for at least 30 days after the last dose of immunotherapy. Multiple events were counted once for each patient and the most severe event was summarized.

Statistical analysis. Data are expressed as the mean \pm standard deviation. For statistical analysis, receiver operating characteristic (ROC) analysis was used to determine the optimal cutoff values for continuous variables. Comparison of non-normally distributed variables between groups was performed using the Mann-Whitney $U$ test. Comparison of categorical variables between two groups was performed using the $\chi^{2}$ test. The probability of survival was estimated with the Kaplan-Meier method, and the differences in survival rates were evaluated by the log-rank test. Multivariate analysis of prognostic factors was conducted using the Cox regression model. All statistical analyses were performed using SPSS v13.0 for Windows (SPSS Inc., Chicago, IL, USA). P $<0.05$ was considered to indicate a statistically significant difference.

\section{Results and Discussion}

Univariate analysis of CD57-related immune cells in the CD8+ T cell differentiation pathway. Cox proportional-hazards regression analysis was conducted to determine 
Table I. Univariate analysis.

\begin{tabular}{lllc}
\hline Groups & HR & HR 95\% CI & P-value \\
\hline CD57+ T cells & 0.362 & $0.186-0.704$ & 0.003 \\
CD8+CD57+ T cells & 0.424 & $0.220-0.820$ & 0.011 \\
Early-CD8+ T cells (CD27+CD8+CD57- T cells) & 5.937 & $2.589-13.6$ & $<0.0001$ \\
Intermediate-CD8+ T cells (CD27+CD8+CD57+ T cells) & 2.325 & $1.216-4.447$ & 0.011 \\
Terminal-CD8+ T cells (CD27-CD8+CD57+ T cells) & 0.154 & $0.072-0.329$ & $<0.0001$ \\
End-CD8+ T cells (CD27-CD8+CD57- T cells) & & & 0.337
\end{tabular}

HR, hazard ratio; CI, confidence interval; $\mathrm{CD}$, cluster of differentiation.

Table II. Multi-variate analysis.

\begin{tabular}{llcc}
\hline Variable & HR & HR 95\% CI & P-value \\
\hline Univariate analysis & & & \\
CD57+ T cells & 0.362 & $0.186-0.704$ & 0.003 \\
CD8+CD57+ T cells & 0.424 & $0.220-0.820$ & 0.011 \\
Early-CD8+ T cells (CD27+CD8+CD57- T cells) & 5.937 & $2.589-13.6$ & $<0.0001$ \\
Intermediate-CD8+ T cells (CD27+CD8+CD57+ T cells) & 2.325 & $1.216-4.447$ & 0.011 \\
Terminal-CD8+ T cells (CD27-CD8+CD57+ T cells) & 0.154 & $0.072-0.329$ & $<0.0001$ \\
CD57 ratio & 0.143 & $0.065-0.314$ & $<0.0001$ \\
Multi-variate analysis & & & $<0.0001$ \\
CD57 ratio & 0.143 & $0.065-0.314$ & \\
\hline
\end{tabular}

$\mathrm{HR}$, hazard ratio; $\mathrm{CI}$, confidence interval; $\mathrm{CD}$, cluster of differentiation.

A

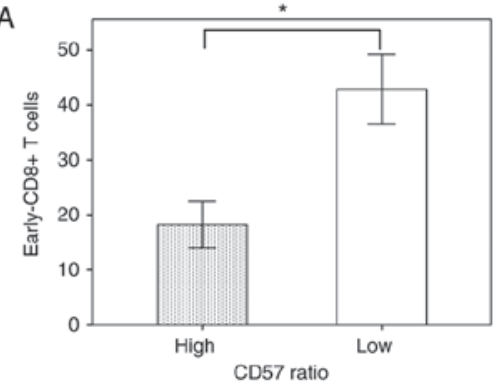

B

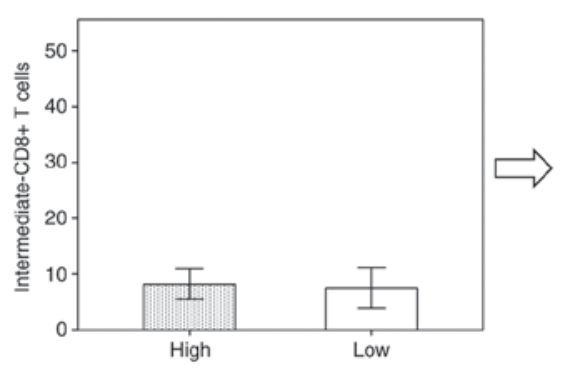

C

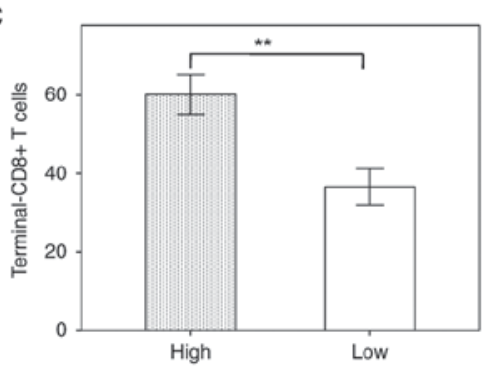

Figure 2. Comparison of the percentage of early-CD8+ T cells (A), intermediate-CD8+ T cells (B), and terminal-CD8+ T cells (C) between patients with high and low CD57 ratios. In the patients with high CD57 ratio, the percentage of terminal-CD8+ T cells is high and that of early-CD8+ T cells low, whereas, in the patients with low CD57 ratio, that of terminal-CD8+ T cells is low and that of early-CD8+ T cells high (t-test $\left.{ }^{*} \mathrm{P}<0.0001,{ }^{* *} \mathrm{P}<0.0001\right)$.

the factors influencing the PFS rate of Stage IV cancer patients (Table I). Based on the univariate analysis of seven variables, early-CD8+ T cells and intermediate-CD8+ T cells were significantly associated with a shorter PFS, whereas CD57+ T cells, CD8+CD57+ T cells, and terminal-CD8+ $\mathrm{T}$ cells were significantly correlated with a longer PFS (Table I). In contrast to the several reports describing an association of CD8+CD57+ $\mathrm{T}$ cells with poor prognosis, in this study, both CD8+CD57+ T cells and CD57+ T cells were significantly associated with a longer PFS (Table I). However, these results are in line with previous reports (6) showing that intermediate-CD8+ $\mathrm{T}$ cells $(\mathrm{CD} 27+\mathrm{CD} 8+\mathrm{CD} 57+$
$\mathrm{T}$ cells) and terminal-CD8+ T cells (CD27-CD8+CD57+ $\mathrm{T}$ cells) were associated with a shorter and a longer PFS, respectively. In addition, in this study, early-CD8+ T cells were more significantly associated with a shorter PFS than were their downstream population of intermediate-CD8+ T cells. To our knowledge, this is the first report showing that early-CD8+ T cells are associated with poor prognosis and may play a major role in establishing the immunosuppressive state of cancer patients.

Early-CD8+ T cells express CD27 differently from terminal-CD8+T cells.CD27 is a member of the tumornecrosis factor-receptor superfamily, acting as a co-stimulatory factor, 
A

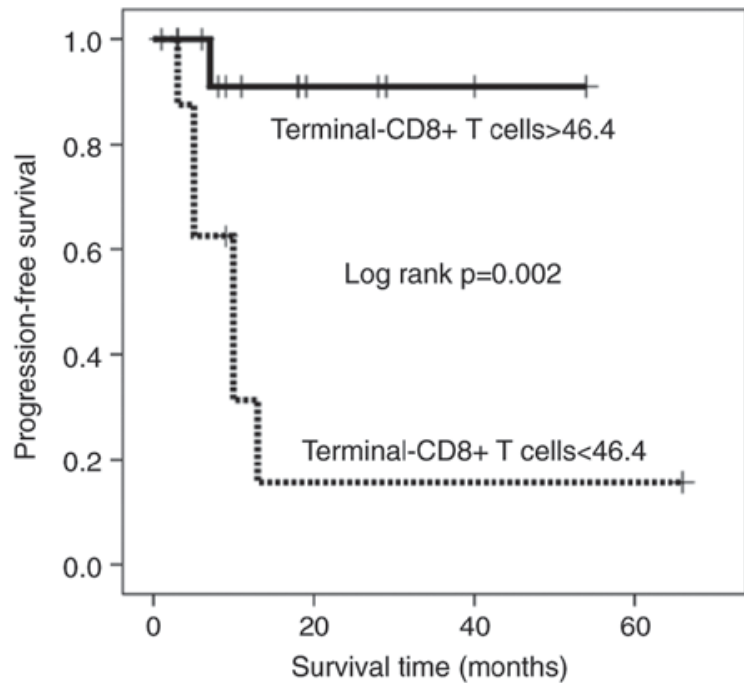

B

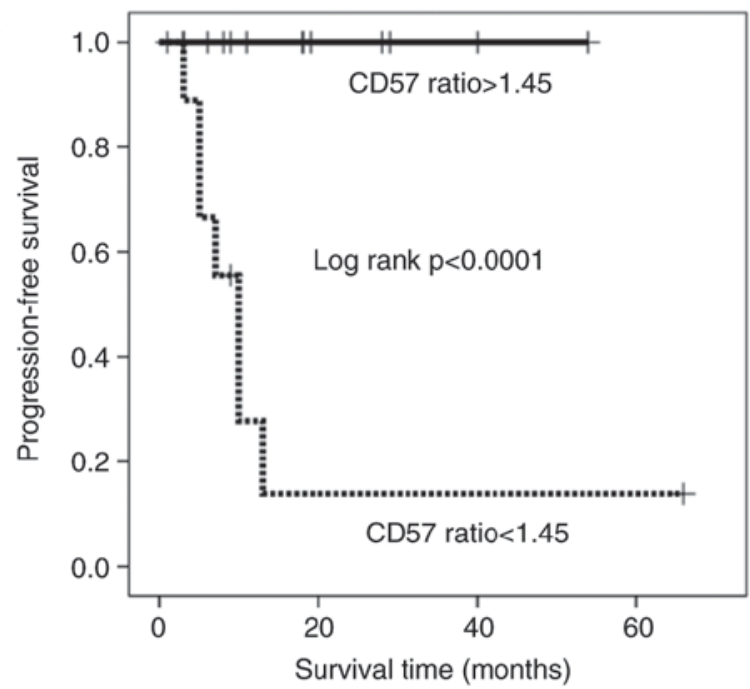

Figure 3. Comparison of progression-free survival between patients with high (bold line) and low (dotted line) percentages of terminal-CD8+ T cells (A) and CD57 ratio (B). The patients with high-terminal-CD8+ T cells (A) and high-CD57 ratio demonstrated a significantly longer survival rate than those with low-terminal-CD8+ T cells and low-CD57 ratio, respectively. Values indicate the cumulative progression-free survival rate.

and CD27 signaling can either improve or suppress $\mathrm{T}$ cell function depending on the level, duration, and timing of the expression of CD27 ligand (CD70) (9-12). CD27 signaling was found to be mediated by constitutive CD70 expression in various cancers $(9,13-14)$, which in turn induces FOXP3+ regulatory $\mathrm{CD} 8+\mathrm{T}$ cells, leading to the immunosuppressive environment characteristic of cancer patients. FOXP3+ regulatory $\mathrm{CD} 8+\mathrm{T}$ cells have also been reported to inhibit CD8+ $\mathrm{T}$ cell differentiation (15), so that early-CD8+ $\mathrm{T}$ cells and terminal-CD8+ T cells are increased and decreased, respectively, which is similar to the situation observed in patients with a low CD57 ratio, as described below. The results of this study suggest that early-CD8+ T cells $(\mathrm{CD} 27+\mathrm{CD} 8+\mathrm{CD} 57-$ $\mathrm{T}$ cells) may play an immunosuppressive role, possibly by inducing FOXP3+ regulatory function. This hypothesis requires further detailed investigation since we did not determine the expression of FOXP3 on the early-CD8+ T cells in the present study.
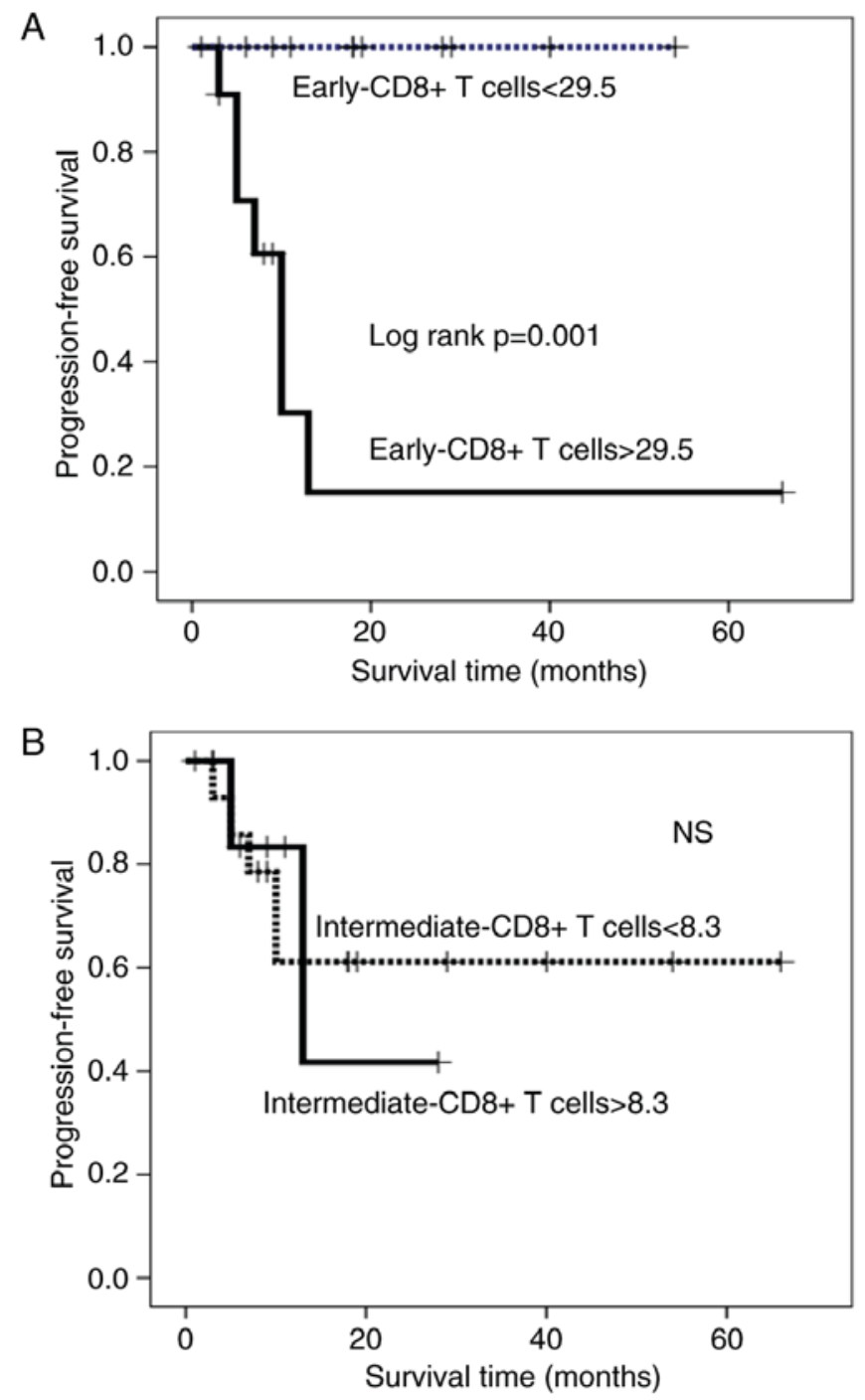

Figure 4. Comparison of progression-free survival between patients with high (bold line) and low (dotted line) percentages of early-CD8+ T cells (A) and intermediate-CD8+ T cells (B). The patients with high-early-CD8+ T cells (A) and high-intermediate-CD8+ T cells demonstrated a significantly shorter survival rate than those with low-early-CD8+ T cells and low-intermediate-CD8+ T cells, respectively. Values indicate the cumulative progression-free survival rate.

CD57 ratio. As shown in Fig. 1, the terminal-CD8+ T cells showed an inverse correlation to the early-CD8+ T cells (Spearman correlation coefficient $-0.919, \mathrm{P}<0.0001$ ). Therefore, to find a more significant prognostic factor, we calculated the CD57 ratio (terminal-CD8+ T cells/early-CD8+ T cells). Indeed, the CD57 ratio was more significantly associated with a longer PFS compared to terminal-CD8 $+\mathrm{T}$ cells (hazard ratio $=0.143$; $95 \%$ confidence interval $=0.065-0.314 ; \mathrm{P}<0.0001)$. In the patients with a high CD57 ratio, early-CD8+ T cells and terminal-CD8+ T cells were significantly decreased and increased, respectively, as compared to those with a low CD57 ratio (Fig. 2A and C), in whom cytotoxic terminal-CD8+ $\mathrm{T}$ cells were predominant. In contrast, in the patients with a low CD57 ratio, early-CD8+ $T$ cells and terminal-CD8+ T cells were significantly increased and decreased, respectively, as compared to those with a high CD57 ratio (Fig. 2A and C), in whom immunosuppressive early-CD8+ $T$ cells were predominant. These results suggest that the CD57 ratio can accurately reflect the degree 

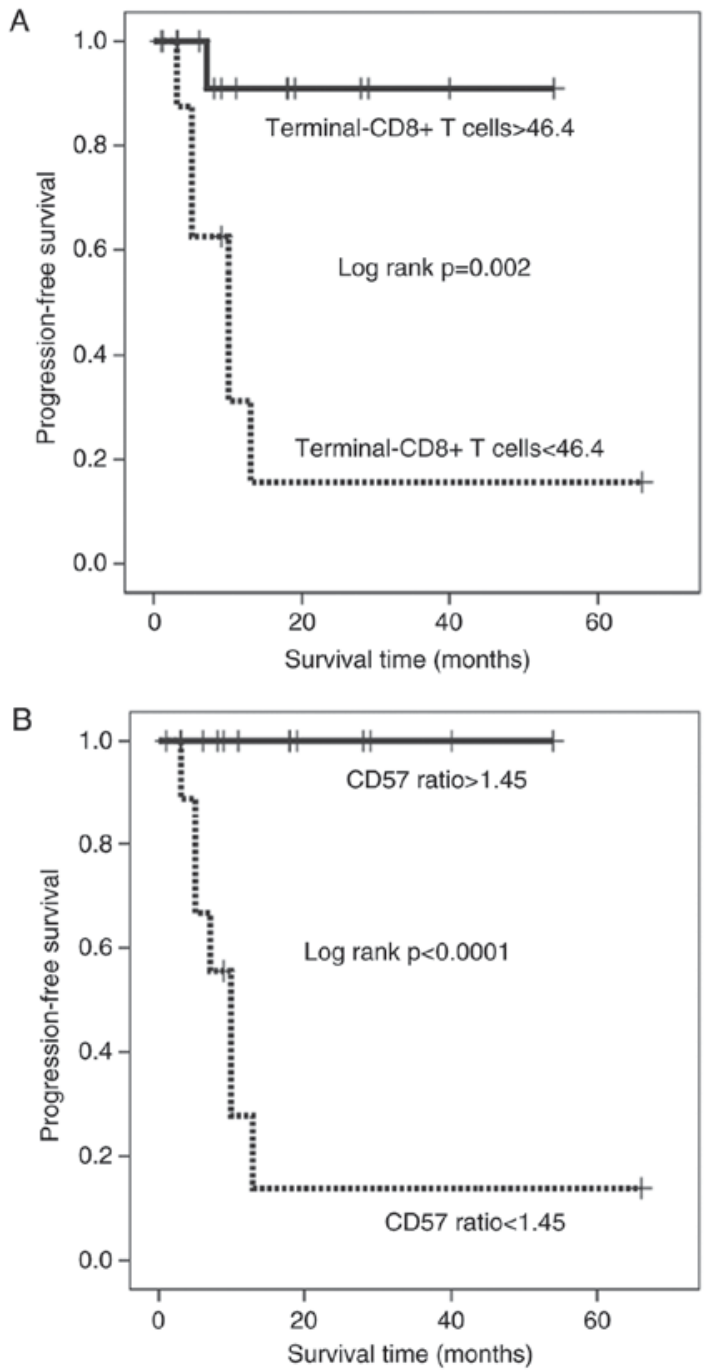

Figure 5. Comparison of progression-free survival between the colon cancer patients with high (bold line) and low (dotted line) percentages of terminal-CD8+ T cells (A) and CD57 ratios (B). The patients with high-terminal-CD8+ T cells (A) and high-CD57 ratio demonstrated a significantly longer survival rate than those with low-terminal-CD8+ T cells and low-CD57 ratio, respectively. Values indicate the cumulative progression-free survival rate.

of predominance of cytotoxic vs. immunosuppressive populations. Thus, we propose that the CD57 ratio can serve as a useful immune parameter to display the dynamic status of CD8+ T cell differentiation.

Multivariate analyses and PFS. To determine the independent value and relative risk of these prognostic factors, we performed a multivariate analysis of the six possible determinants (CD57+ T cells, CD8+CD57+ T cells, early-CD8+ T cells, intermediate-CD8+T cells, terminal-CD8+Tcells, and CD57 ratio) using the Cox regression model. The results confirmed that the CD57 ratio was an independent PFS predictor of Stage IV cancer patients (Table II).

The univariate analysis results showed that the proportions of early-CD8+ T cells, intermediate-CD8+ T cells, terminal-CD8+ $\mathrm{T}$ cells, and the CD57 ratio had a more significant influence on the PFS rate than the other possible determinants, but not on the Overall Survival (OS). We
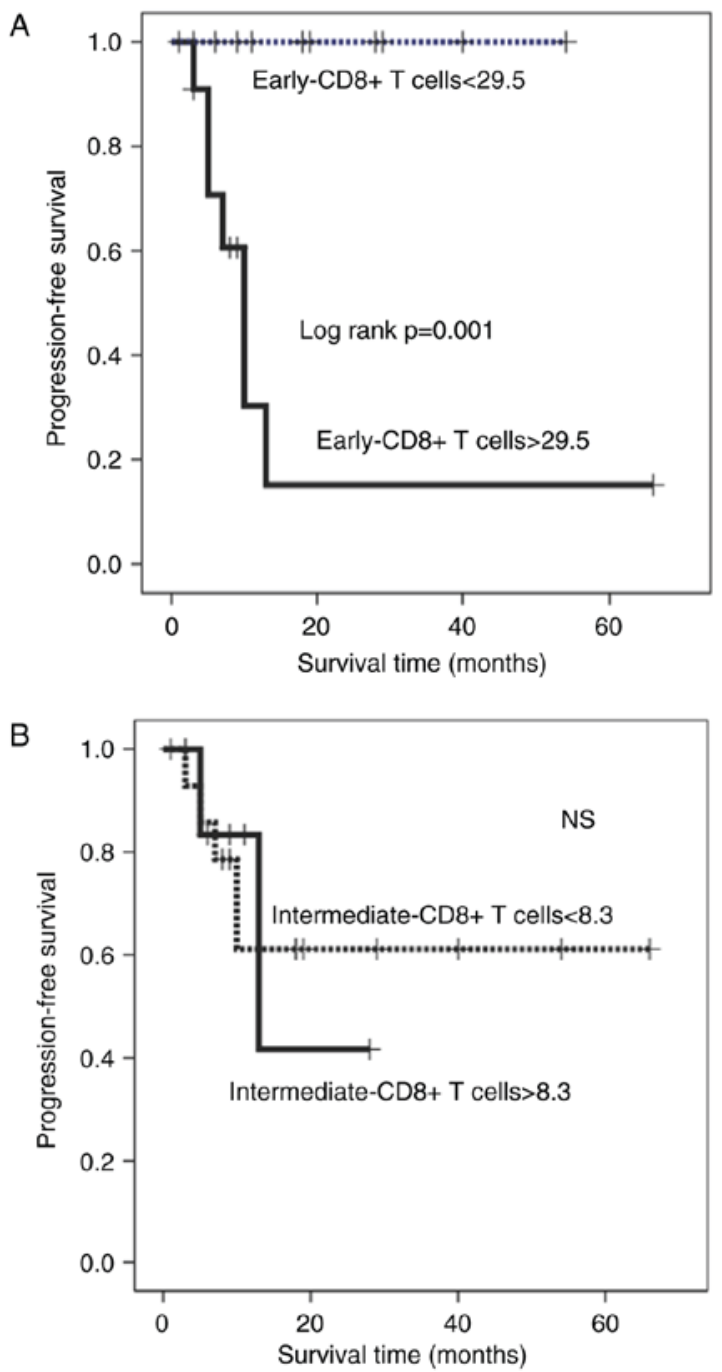

Figure 6. Comparison of progression-free survival between the colon patients with high (bold line) and low (dotted line) percentages of early-CD8+ T cells (A) and intermediate-CD8+ T cells (B). The patients with high-early-CD8+ T cells (A) demonstrated a significantly shorter survival rate than those with low-early-CD8+ T cells. Values indicate the cumulative progression-free survival rate.

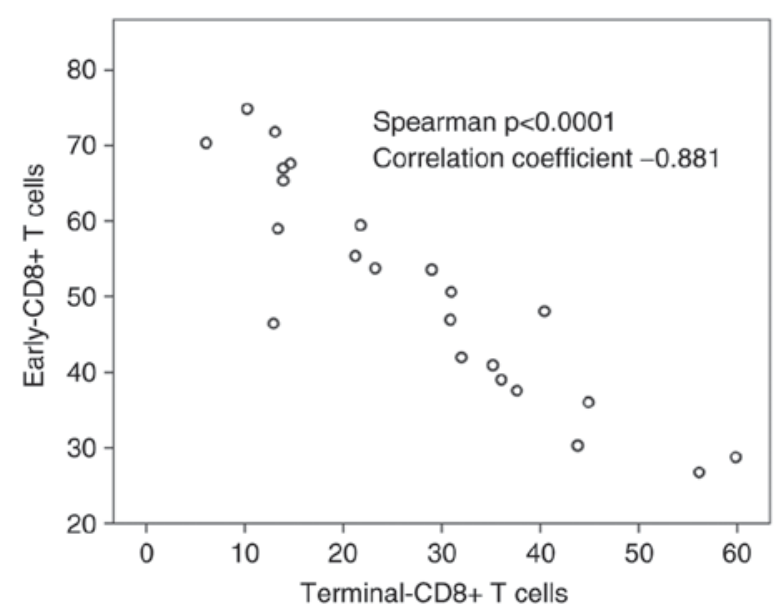

Figure 7. Correlation between early-CD8+ T cells and terminal-CD8+ $\mathrm{T}$ cells in the peripheral blood of colon cancer patients. There is a significant reverse correlation between them (Spearman correlation coefficient $=-0.881$, $\mathrm{P}<0.0001)$ 


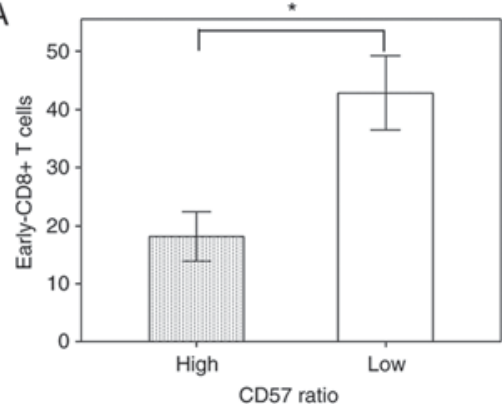

B

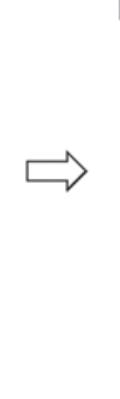

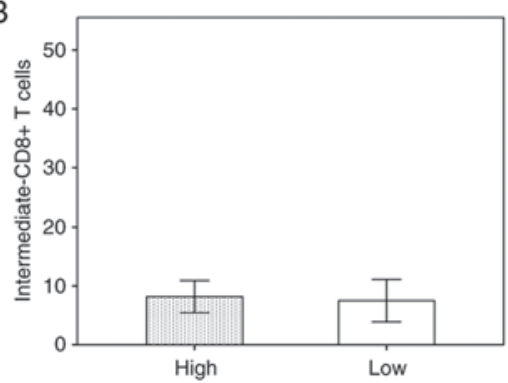

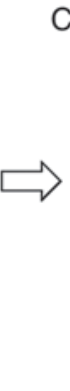

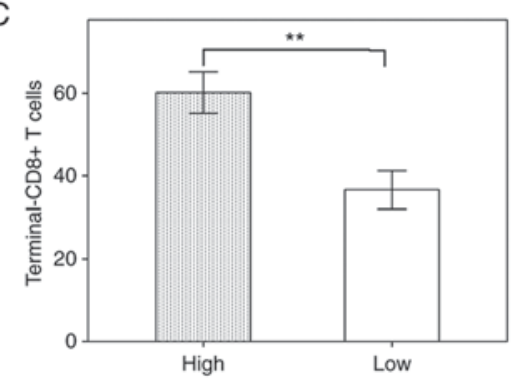

Figure 8. Comparison of the percentage of early-CD8+ T cells (A), intermediate-CD8+ T cells (B), and terminal-CD8+ T cells (C) between colon cancer patients with high and low CD57 ratios. In the colon cancer patients with high CD57 ratio, the percentage of terminal-CD8+ T cells is high and that of early-CD8+ T cells is low, whereas, in the colon cancer patients with low CD57 ratio, that of terminal-CD8+ T cells is low and that of early-CD8+ T cells is high (t-test ${ }^{*} \mathrm{P}<0.0001,{ }^{* *} \mathrm{P}<0.0001$ ).

then divided the Stage IV cancer patients into groups based on the proportions of each of these markers with cutoff values of $19.1,8.3,33.7$, and $21.8 \%$, respectively, as determined using the ROC curve (sensitivity of $80,54.1$, 68 , and $88.9 \%$; specificity of $68,71.4,100$, and $75 \%$; area under the curve of $0.748,0.587,0.788$, and $0.819 ; \mathrm{P}=0.024$, $0.146,0.009$, and 0.005, respectively). The Kaplan-Meier survival curve revealed that patients with a high percentage of terminal-CD8+ T cells and those with a high CD57 ratio had a significantly longer PFS duration than those with a low percentage of terminal-CD8+ T cells and those with a low CD57 ratio, respectively (Kaplan-Meyer log-rank $\mathrm{P}<0.0001$; Fig. $3 \mathrm{~A}$ and $\mathrm{B}$ ). In contrast, patients with higher percentages of early- and intermediate-CD8+ T cells had a significantly shorter PFS duration than those with lower percentages (Kaplan-Meyer log-rank $\mathrm{P}<0.0001$; Fig. 4A and B). The median of PFS was 10 months in the patients with low-CD57 ratio, 8 months in the patients with low-terminal-CD8+ T cells, and 11 months in the patients with low-early-CD8+ T cells. However, the two-year survival rate of patients with a high percentage of early- and intermediate-CD8+ $\mathrm{T}$ cells was 11.1 and $28.1 \%$, respectively, suggesting that early-CD8 $+\mathrm{T}$ cells are more strongly associated with a state of immunosuppression in Stage IV cancer patients than are intermediate-CD8+ T cells. These results that terminal-CD8+ $\mathrm{T}$ cells and early-CD8+ $\mathrm{T}$ cells may have cytotoxic and immunosuppressive function, respectively, are only preliminary data, so that we should perform further experiments to examine the expression of cytotoxic markers in terminal-CD8 $+\mathrm{T}$ cells and of immunosuppressive markers such as PD-1 and FOXP3 in early-CD8+ T cells.

The results in this study obtained from the cancer patients with different origin may be deficient in credibility because tumors with different origin have very different biology. We selected the 23 patients with colon carcinomas from all patients and performed the same analysis as to PFS. The Kaplan-Meier survival curve revealed that patients with a high percentage of terminal-CD8 + T cells and those with a high CD57 ratio had a significantly longer PFS duration than those with a low percentage of terminal-CD8+ T cells and those with a low CD57 ratio, respectively (Kaplan-Meyer log-rank $\mathrm{P}=0.002$ and $\mathrm{P}<0.0001$; Fig. 5A and $\mathrm{B})$. In contrast, patients with higher percentages of early-CD8+ $\mathrm{T}$ cells had a significantly shorter PFS duration than those with lower percentages (Kaplan-Meyer log-rank $\mathrm{P}=0.001$; Fig. 6A). Terminal-CD8+ T cells showed a significant reverse correlation to early-CD8+ $\mathrm{T}$ cells (correlation coefficient=-0.881 Spearman $\mathrm{P}<0.0001)$ (Fig. 7). The percentage of terminal-CD8+ $\mathrm{T}$ cells was higher in the patients with high-CD57 ratio than in those with low-CD57 ratio, whereas the percentage of early-CD8+ T cells was higher in the patients with low-CD57 ratio than in those with high-CD57 ratio (Fig. 8). These results obtained from the colon cancer patients also lead to the same conclusion that the CD57 ratio appears to be the most powerful immunological prognostic parameter obtained from the peripheral blood, precisely reflecting the state of CD8+ $\mathrm{T}$ cell-differentiation.

Our results suggest that the CD57 ratio is a convenient and powerful marker for predicting the prognosis of Stage IV cancer patients given that it was a better independent prognostic factor than terminal-CD8+ T cells, i.e., cytotoxic effector cells, and is easily calculated by the data obtained from peripheral blood samples. Further, the CD57 ratio could predict the dynamic state of $\mathrm{CD} 8+\mathrm{T}$ cell differentiation (measurement of the predominance of cytotoxic vs. immunosuppressive CD8+ T cells). We also revealed that early-CD8+ $\mathrm{T}$ cells (CD27+CD8+CD57- $\mathrm{T}$ cells) are the more important immunosuppressive population in patients with Stage IV carcinomas compared to intermediate-CD8+ T cells, which are only preliminary data, so that we should perform further experiments to examine the expression of immunosuppressive markers such as PD-1 and FOXP3 in early-CD8+ T cells. The adoption of these markers can help to improve personalized medicine for late-stage cancer patients, leading to their optimum treatment.

\section{References}

1. Akagi J and Baba H: Prognostic value of CD57(+) T lymphocytes in the peripheral blood of patients with advanced gastric cancer. Int J Clin Oncol 13: 528-535, 2008.

2. Characiejus D, Pasukoniene V, Kazlauskaite N, Valuckas KP, Petraitis T, Mauricas M and Den Otter W: Predictive value of CD8highCD57+ lymphocyte subset in interferon therapy of patients with renal cell carcinoma. Anticancer Res 22: 3679-3683, 2002. 
3. Focosi D, Bestagno M, Burrone O and Petrini M: CD57+ T lymphocytes and functional immune deficiency. J Leuloc Biol 87: 107-116, 2010.

4. Dondi E, Roué G, Yuste VJ, Susin SA and Pellegrini S: A dual role of IFN-alpha in the balance between proliferation and death of human CD4+ T lymphocytes during primary response. J Immunol 173: 3740-3747, 2004.

5. Characiejus D, Pasukoniene V, Jonusauskaite R, Azlauskaite N, Aleknavicius E, Mauricas M and Otter WD: Peripheral blood CD8high CD57+ lymphocyte levels may predict outcome in melanoma patients treated with adjuvant interferon-alpha. Anticancer Res 28: 1139-1142, 2008.

6. Wu RC, Hwu P and Radvanyi LG: New insights on the role of CD8(+)CD57(+) T-cells in cancer. Oncoimmunology 1: 954-956, 2012.

7. Brenchley JM, Karandikar NJ, Betts MR, Ambrozak DR, Hill BJ, Crotty LE, Casazza JP, Kuruppu J, Migueles SA, Connors M, et al: Expression of CD57 defines replicative senescence and antigen-induced apoptotic death of CD8+ T cells. Blood 101: 2711-2720, 2003.

8. Sobin LH and Wittekind CH: UICC TNM Classification of malignant tumours. John Wiley and Sons, New York, 1997.

9. Papagno L, Spina CA, Marchant A, Salio M, Rufer N, Little S, Dong T, Chesney G, Waters A, Easterbrook P, et al: Immune activation and $\mathrm{CD} 8+\mathrm{T}$-cell differentiation towards senescence in HIV-1 infection. PLoS Biol 2: E20, 2004.
10. Nolte MA, van Olffen RW, van Gisbergen KP and van Lier RA: Timing and tuning of CD27-CD70 interactions: The impact of signal strength in setting the balance between adaptive responses and immunopathology. Immunol Rev 229: 216-231, 2009.

11. Matter M,Odermatt B, Yagita H, Nuoffer JM and Ochsenbein AF: Elimination of chronic viral infection by blocking CD27 signaling. J Exp Med 203: 2145-2155, 2006.

12. Penaloza-MacMaster P, Ur Rasheed A, Iyer SS, Yagita H, Blazar BR and Ahmed R: Opposing effects of CD70 costimulation during acute and chronic lymphocytic choriomeningitis virus infection of mice. J Virol 85: 6168-6174, 2011.

13. Borst J, Hendriks J and Xiao Y: CD27 and CD70 in T cell and B cell activation. Curr Opin Immunol 17: 275-281, 2005.

14. Wischhusen J, Jung G, Radovanovic I, Beier C, Steinbach JP, Rimner A, Huang H, Schulz JB, Ohgaki H, Aguzzi A, et al: Identification of CD70-mediated apoptosis of immune effector cells as a novel immune escape pathway of human glioblastoma. Cancer Res 62: 2592-2599, 2002.

15. Claus C, Riether C, Schürch C, Matter MS, Hilmenyuk T and Ochsenbein AF: CD27 signaling increases the frequency of regulatory $\mathrm{T}$ cells and promotes tumor growth. Cancer Res 72 : 3664-3676, 2012. 